\title{
Probing Nanostructures in Food with the AFM.
}

\author{
Morris VJ \\ Institute of Food Research, Norwich Research Park, Colney, Norwich NR4 7UA, UK. \\ [e-mail: vic morris@bbsrc.ac.uk].
}

\begin{abstract}
The atomic force microscope (AFM) provides a tool for probing complex food structures at the molecular level. The advantages of the AFM over electron microscopy are minimal sample preparation, the ability to image in liquid or gaseous environments and novel contrast mechanisms, which provide unique insights into food structures. The ability to visualise the nanostructures present in foods has led to the solution to previously intractable problems in food science. This will be illustrated through research studies on the factors affecting the stability of foams and emulsions and the functional properties of starch.
\end{abstract}

Keywords: AFM, atomic force microscopy, interfaces, competitive displacement, foams emulsions, starch.

\section{INTRODUCTION}

The atomic force microscope provides a tool for probing the molecular structure underlying the functional properties of food systems. The method of imaging permits the AFM to investigate molecular structure and molecular interactions under 'near-native' conditions. In addition the AFM provides new contrast mechanisms which provide new information on the structure of food systems. Over the last 15 years the use of probe microscopy has led to the solution to previously intractable problems in food science. The use of this type of approach will be illustrated through research studies on two areas of importance in food science: the stability of food foams and emulsions and the structure and function of starch.

Food foams and emulsions: The preparation of a food foam or emulsion requires the creation and the stabilisation of an air-water or oil-water interface. The types of interfaces encountered in food systems will contain a wide range of surface-active molecules. The structures formed by the molecules and the interactions between these components will determine the long-term stability of the foam or emulsion. The most common types of species present at interfaces in foods will be proteins and various small, highly mobile molecules such as surfactants or lipids. In addition a small number of polysaccharides are known to be surface-active. The majority of food foams and emulsions will be stabilized by proteins and/or surfactants. Proteins and surfactants (or lipids) are capable, on their own, of stabilising interfaces, but they do so by different molecular mechanisms (Wilde et al, 2004).

Proteins are considered to adsorb at the interface, to partially unfold and then to interact to form molecular 'gel-like' networks (Wilde et al, 2004). Collapse of emulsions or foams involves the stretching of the interface and the elasticity of the protein structure is supposed to oppose this effect. In the case of mobile surfactants (or lipids) stretching of the interface will lead to concentration gradients and rapid diffusion of the molecules to restore the status quo (Wilde et al, 2004). 
A source of instability for most foods is the presence of both proteins and small mobile molecules at the interface. The incompatibility of the two stabilisation mechanisms means that the mixed interfaces are less stable than 'pure' interfaces, which contain just protein or surfactant (or lipid) (Wilde et al, 2004). Provided there is sufficient surfactant (or lipid) present then these molecules will eventually displace the protein. It is the structures formed during this battle for control of the interface that give rise to instability in the food foam or emulsion.

So, how do small mobile surfactant molecules competitively displace proteins? The answer to this question has been obtained by visualising the structural changes that occur during the displacement using probe microscopes such as the atomic force microscope. Understanding the interactions that occur at an interface that contains just one type of protein and one type of surfactant is of importance, but is not the whole story. In real food systems there will almost always be mixtures of proteins that will be present at the interface, and it is necessary to understand what sorts of structures are formed by these mixtures of proteins, and how they resist displacement. An understanding of the role of proteins at interfaces provides clues to the functional behaviour of surface-active polysaccharides. In general the surface-activity is usually attributed to the presence of protein and the 'polysaccharide' is believed to be a protein-polysaccharide complex. Understanding the nature of this complex provides a mechanism for explaining and optimising surface activity.

Starch: The currently accepted model for the structure of starch granules is described in recent review articles (Gallant et al, 1997; Buléon et al, 1998). The starch granules are pictured as semi-crystalline spheroidal structures containing concentric layers of 'crystalline' and 'amorphous' growth rings. Gallant and coworkers (1997) have revived and redefined the 'blocklet model' of starch ultrastructure, in which blocklets are considered to be essentially parcels of the partially-crystalline starch polysaccharide amylopectin, contained within the growth ring structure of the starch granule. The crystalline component of the blocklets arises from the ordering of the short branches of the amylopectin molecules. In order to account for factors such as the dependence of the level of crystallinity on the degree of hydration of the starch, it has been proposed that the organisation of the amylopectin branches can be redefined in terms of a 'side-chain liquid-crystalline model' for starch (Waigh et al, 1998; 2000). The location of the linear amylose molecules is more difficult to determine. However, in general the amylose molecules are believed to occupy the regions surrounding the blocklets, although a percentage of the amylose molecules may traverse blocklets, and the regions within the granule which contain amylose are generally considered to be amorphous.

The recent evidence in support of a blocklet model has come largely from electron microscopy (Helbert \& Chanzy, 1996; Gallant et al, 1997). Atomic force microscopy provides an ideal tool for probing granule ultrastructure. However, little is known about the origins of contrast in AFM images of starch. With a new technique such as AFM it is important to identify and understand the origin of contrast in order to be able to interpret AFM images. This article describes experiments designed to identify the origins of contrast in AFM images of starch, and the use of AFM to understand how starch structure can be modified to change the functional and nutritional properties of the starch.

\section{ATOMIC FORCE MICROSCOPY}

AFMs are the most widely used form of probe microscope in food science (figure 1). They have proved particularly useful for solving previously intractable problems relating to the 
molecular structure of foods (Morris, 2004). The AFM generates images by 'feeling' rather than 'looking' at the sample. In general the sample is scanned beneath a sharp probe which is attached to a cantilever. Any changes in the force between the sample and the probe will cause the cantilever to deflect and these deflections are monitored using an optical lever: a low-power laser beam directed onto the end of the cantilever. The positional change of the reflected laser beam is detected by monitoring its position on the surface of a four-quadrant photodiode. Hence the deflection of the cantilever is measured as a potential difference. In the normal mode of operation of the AFM this change in deflection of the cantilever at each sample point, the so-called error signal, is utilised via a feed-back circuit, to adjust the sample-probe separation, in order to maintain a constant, preset cantilever deflection. Thus a 3D topographic image can be generated by amplifying the resultant changes in sample-tip separation. Because the probe is effectively touching the sample this type of imaging mode is termed 'contact mode imaging'. If the sample is homogeneous then imaging at constant cantilever deflection is the same as imaging at constant applied force. The force changes are entirely due to the changes in probe-sample separation during scanning and the resultant image is a true representation of surface topography. If the sample is heterogeneous then other factors will influence the change in force during scanning and hence alter contrast in the image. A variety of additional imaging modes are available which utilise this fact and can be used to emphasise molecular structure, or to probe structural features such as charge or elasticity. The principle of operation allows the AFM to be operated in air or under liquids. The operating range spans that accessible to both the light and the electron microscopes, allowing molecular resolution under more natural conditions.

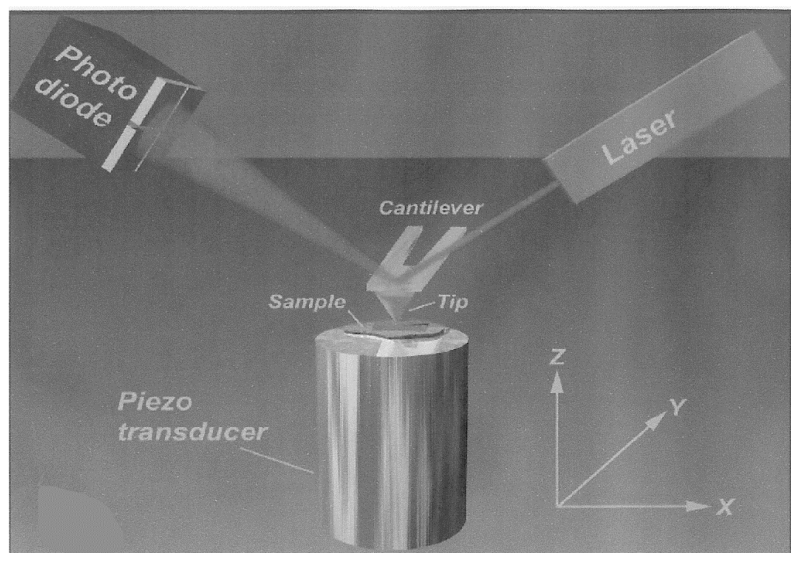

Figure 1. Schematic diagram showing the operating principle of an atomic force microscope.

\section{FOOD FOAMS AND EMULSIONS}

Sample preparation: In order to image the interfacial structures found in food foams and emulsions by AFM it is necessary to model these structures on a Langmuir trough and sample the interfacial structure using Langmuir-Blodgett (LB) methods (Gunning et al, 1996; Mackie et al, 1999). Samples for AFM are collected on mica substrates and imaged under 1-butanol, in order to control the applied force: for the samples described the AFM images were obtained in the dc contact mode using minimal applied force (Gunning et al, 1996; Mackie et al, 1999). Mixed interfaces can be generated by spreading or co-adsorbing the molecules. Both air-water and oil-water interfaces can be investigated using this approach (Mackie et al, 1999; 2000a). Sampling the interfacial structures for spread films is comparatively simple. However, the procedures need to be modified when co-adsorbed films are prepared, in order 
to prevent any passive adsorption of bulk protein during the LB dip, which would otherwise obscure the structure of the interfacial film (Mackie et al, 1999).

Protein-surfactant interactions: The simplest type of mixed interface that can be studied consists of a protein and a small molecule, such as a surfactant. This can be used to model the competitive displacement of proteins by surfactants. Figure 2 displays a series of AFM images which show the competitive displacement of a spread $\beta$-lactoglobulin film from an air-water interface by the non-ionic surfactant Tween 20. The contrast (grey levels) in the image is determined by the height of the sample above the substrate. Bright regions represent protein and the black areas show where the surfactant has invaded the interface: for Tweens the 1-butanol removes the surfactant, effectively 'fixing' the structure. The images (figures $2 \mathrm{a}-\mathrm{d}$ ) correspond to a progressive increase in surface pressure and show increased coverage of the interface with surfactant. These images clearly confirm that the proteins form networks at the interface (Mackie et al, 1999).

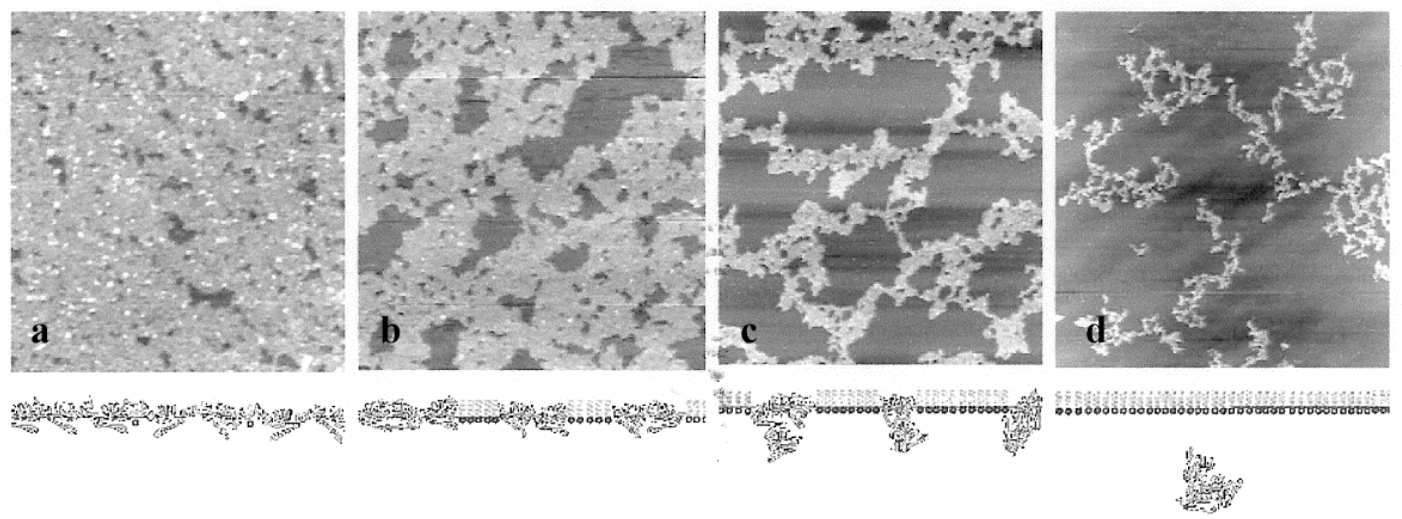

Figure 2. A series of AFM images which show the competitive displacement of a spread protein ( $\beta$ lactoglobulin) film from an air-water interface by the progressive addition of the surfactant (polyoxyethylene sorbitan monolaurate) Tween 20. The image sizes are (a) $1.0 \times 1.0 \mu \mathrm{m}$, (b) $1.6 \times 1.6 \mu \mathrm{m}$, (c) $3.2 \times 3.2 \mu \mathrm{m}$ and (d) $10.0 \times 10.0 \mu \mathrm{m}$. The cartoon shown beneath the images represents the deduced displacement process.

At the lowest surface pressures it appears that the surfactant is randomly adsorbed onto the interface where small holes have appeared in the protein network. With increasing surface pressure these holes are seen to expand into larger domains until, eventually, at the very highest surface pressures the protein network snaps, leaving protein aggregates abandoned in a sea of surfactant. Purely visual inspection of the images would seem to be compatible with the more surface-active surfactant simply displacing individual proteins at the boundary between protein and surfactant regions.

However, it is possible to quantify the AFM images (Mackie et al, 1999). Areas occupied by either protein or surfactant can be calculated and, when combined with measurements of the thickness of the protein network, the protein volume can be determined. Figure 3 shows the true nature of the displacement process. Protein volume remains constant until the network snaps, showing that no protein is lost from the interface into the bulk until the protein network has been broken. The molecular mechanism of displacement is illustrated as a cartoon in figure 2. Nucleation of surfactant domains occurs at defects in the protein network. The surfactant domains expand and, as the area occupied by protein decreases, the protein layer thickens, keeping the total protein volume constant. Initially this involves refolding of individual proteins, and then buckling of the protein network. Eventually the protein film is pulled out into thin filaments that finally snap, freeing protein and allowing it to be displaced 
into the bulk phase. The mechanism involves folding and buckling of the interfacial layer and has thus been termed (Mackie et al, 1999) an 'orogenic displacement mechanism'.

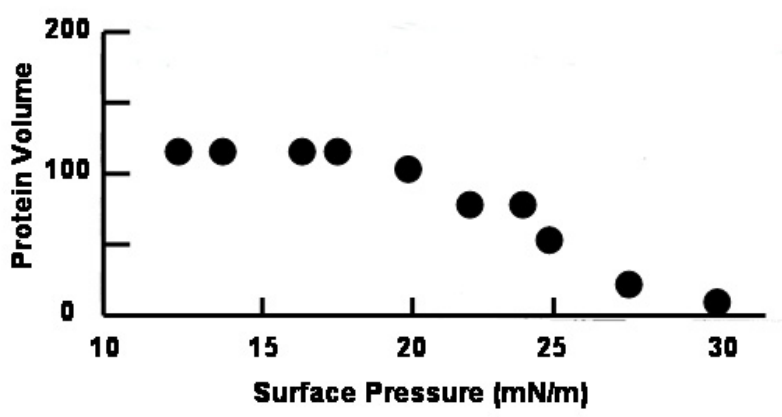

Figure 3. The changes in protein volume during the displacement of a spread $\beta$-lactoglobulin from an airwater interface by the surfactant Tween 20.

The mechanism has been shown to be generic for all proteins and surfactants studied to date (Mackie et al, 1999; Gunning et al, 1999; Mackie et al, 2000a; 200b; 2001a; Gunning et al, 2004). Displacement is orogenic for water-soluble, oil-soluble, neutral and charged surfactants. Similar results are found for spread and co-adsorbed films and for air-water, oilwater and even solid-water interfaces. The mechanism is generic because proteins that stabilise foams or emulsions link together to form networks. Once a network is formed it has to be broken before the protein can be displaced into the bulk.

The AFM led to the discovery of this new displacement mechanism because it allowed visualisation of the interfacial structure at the molecular scale. Heterogeneity of the interfacial structure was crucial to understanding displacement. When proteins are spread at interfaces and then imaged the film is found to contain holes. This is because as proteins adsorb they partially unfold and stick together. As more proteins adsorb onto the interface the space available decreases, and the opportunity to unfold, and to attach to other proteins decreases. The last proteins to arrive are probably passively adsorbed and weakly, if at all, attached to the network. These proteins are easily lost on sampling the interface and are thus likely to be the holes seen in the protein network. Such holes are believed to be the sites that the surfactant can attack allowing invasion of the interface. The boundaries of the expanding surfactant domains are ragged. This is also due to molecular heterogeneity. Individual proteins will unfold and interact to different extents and, as the protein network is compressed, refolding will occur more easily in certain regions, allowing stress to be redistributed around the boundary of the growing domains.

Some small differences are seen for certain proteins. The milk protein $\beta$-casein is a poor foam stabiliser. On displacement with Tween 20 the domain boundaries are circular (Mackie et al, 1999), demonstrating uniform compression and weak inter-protein interactions. However, at oil-water interfaces $\beta$-casein is more difficult to displace (Mackie et al, 2000a). This is consistent with it being better at stabilising emulsions: the domain boundaries are ragged and the network fails at higher surface pressures (Wilde et al, 2004).

The displacement mechanisms for charged surfactants such as cetyl-trimethyl-ammonium bromide (CTAB), sodium dodecyl sulphate (SDS) and lyso-phosphatidylcholinelauroyl (LPC-L) are orogenic, but slightly different (Mackie et al, 2000b; Gunning et al, 2004a; 2004b). SDS initially nucleates into small domains but, with increasing surface pressure, the 
number of nuclei increase but do not expand and grow into larger domains. At the highest surface pressures coalescence of domains appears to occur, just prior to the collapse point of the network (Mackie et al, 200b). For anionic, cationic or zwitterionic surfactants the behaviour is similar (Gunning et al, 2004a; 2004b); displacement involving nucleation but little domain growth. Screening the charge on the surfactant permits growth of surfactant domains: strong charge-charge repulsions between surfactants and localisation of counterions would favour even dispersal of the surfactant across the interface and the presence of many small domains. When the charge is screened then domain growth can arise (Gunning et al, 2004b). For ionic surfactants failure of the protein network occurs at different surface pressures for different surfactants (Gunning et al, 2004b). For CTAB, SDS and LPC-L the failure is found to occur at higher surface pressures than that seen for the neutral Tweens. The binding of ionic surfactants to the proteins alters the failure strength of the network. In fact it can be shown that, at low LPC-L surfactant concentrations, co-adsorption of the proteinsurfactant complex actually leads to an increase in the shear modulus of the protein network (Gunning et al, 2004b), although at higher surfactant concentrations the orogenic mechanism predominates weakening the protein network. Thus the failure occurs at higher than expected surface pressures (Gunning et al, 2004b) revealing potential synergistic effects in mixed ionic and non-ionic surfactants.
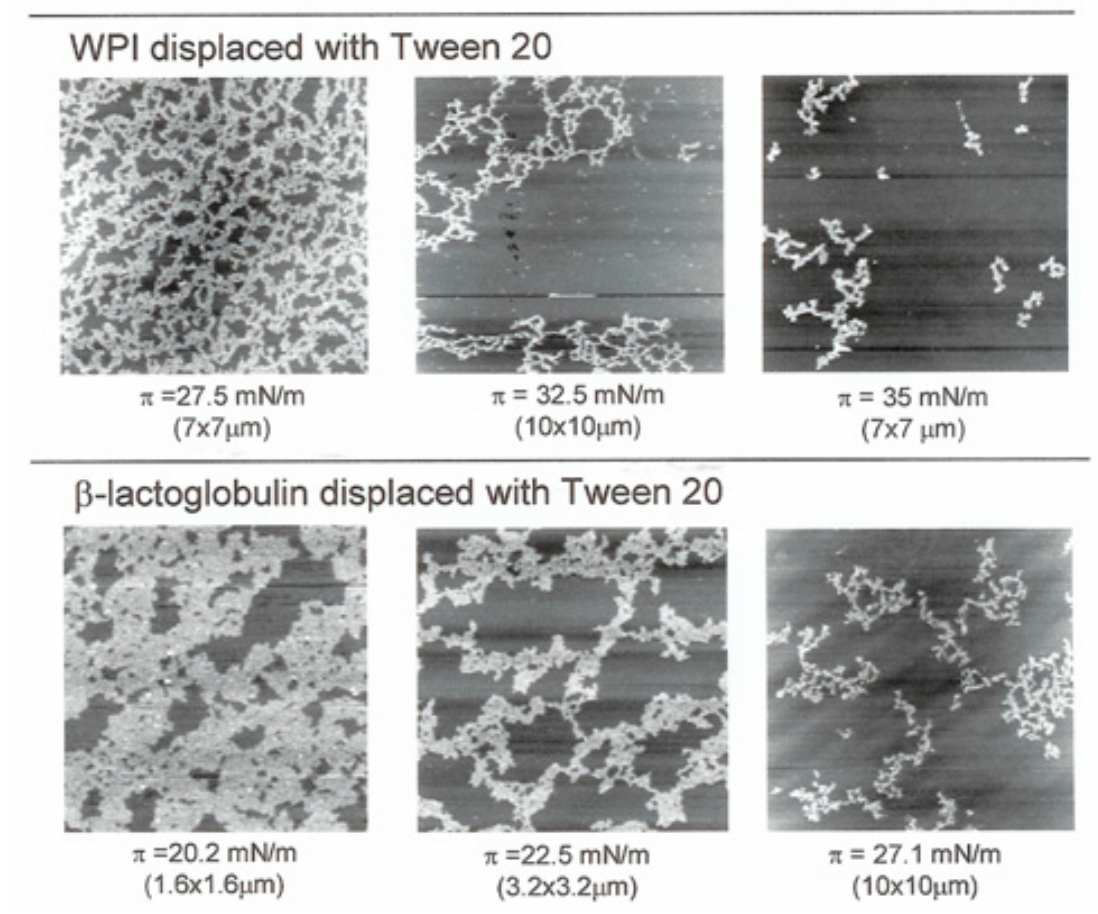

Figure 4. Competitive displacement of spread films of whey protein isolate (WPI) and $\beta$-lactoglobulin from an air-water interface with the non-ionic surfactant Tween 20. The WPI network remains intact at surface pressures $(\pi)$ above those at which pure $\beta$-lactoglobulin networks fail.

Multi-component interfaces: The majority of interfaces encountered in food systems will contain more than one protein. Commercial materials used to stabilise emulsions or foams are complex isolates rather than purified single proteins. In order to describe the behaviour of protein isolates it is necessary to understand the types of structures formed by mixtures of proteins at the interface, and also how such mixed structures are displaced by surfactants.

Most bulk mixtures of native proteins will phase separate. It is constructive to consider whether such phase separation will occur at the interface? As proteins adsorb at the interface 
they partially unfold and stick to one another. If the adsorption is random and the proteins collide and stick together very rapidly then one might expect that the network formed would be an intimately mixed structure. But what type of interface is formed at the interface? Use of fluorescent labelling and visualisation of the mixed networks by both far-field and near-field fluorescent microscopy, suggests that simple binary mixtures of common food proteins show no evidence for gross phase separation (Gunning et al, 2001; Mackie et al, 2001a). The interactions between proteins appear to be rapid and thus kinetically lock the network into an intimately mixed structure. Displacement studies (Gunning et al, 2001; Mackie et al, 2001a) show that, provided there are sufficient concentrations of both proteins present at the interface, the behaviour of simple binary mixtures is related to the behaviour of the pure components. Failure of the mixed network occurs at surface pressures equal to, or less than, that of the component protein which alone most resists displacement. Although more needs to be done on these mixed systems, it does appear that they are likely to behave as simple mixtures, and that synergisms between proteins may be rare.

Protein isolates: The behaviour of simple binary mixtures provides a basis for analysing the behaviour of protein isolates. Whey protein isolate (WPI) contains the milk proteins $\beta$ lactoglobulin, $\alpha$-lactalbumin and bovine serum albumen. The displacement of each of the individual proteins has been studied (Mackie et al, 1999; Gunning et al, 2001). Given that the major component of WPI is $\beta$-lactoglobulin it might be expected that competitive displacement of WPI would resemble that of $\beta$-lactoglobulin. The displacement of WPI from air-water interfaces by Tweens occurs via an orogenic mechanism (figure 4) but the surface pressure at which the network fails is higher than that seen for $\beta$-lactoglobulin alone (Woodward et al, 2004). In this case the difference can be shown to be due to the different conditions under which individual milk proteins are extracted and purified, and the isolation conditions used to prepare WPI. It has been possible to identify the chemical origins of the improved stability of the WPI. Clearly this knowledge can be used to manipulate rationally the properties of WPI.
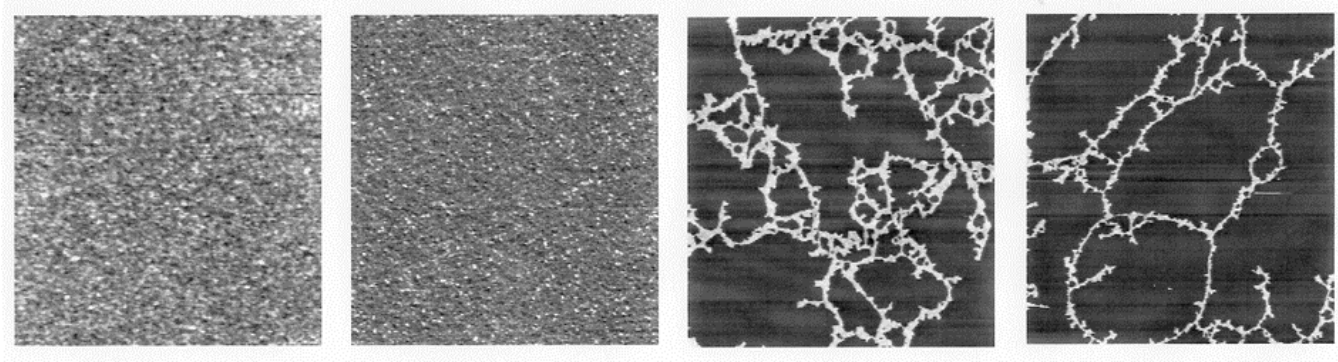

Figure 5. AFM images of the displacement of a spread sodium caseinate layer from an air-water by Tween 20. The image sizes and surface pressures $(\pi)$ are from left-to-right $1.6 \times 1.6 \mu \mathrm{m} \mathrm{\&} 18.4 \mathrm{mN} / \mathrm{m}, 10$ x $10 \mu \mathrm{m} \& 21 \mathrm{mN} / \mathrm{m}, 10 \times 10 \mu \mathrm{m} \& 23 \mathrm{mN} / \mathrm{m}$ and $10 \times 10 \mu \mathrm{m} \& 25 \mathrm{mN} / \mathrm{m}$.

Sodium caseinate is predominately $\beta$-casein with smaller amounts of the $\alpha$-and $\kappa$-caseins. Given that $\beta$-casein is easily displaced from an air-water interface one might expect the same to be true for sodium caseinate. In fact sodium caseinate resists displacement to surface pressures similar to that seen for $\beta$-lactoglobulin; a good foam stabiliser. The structure of the final network prior to failure (figure 5) is atypical of that seen for $\beta$-casein and resembles the network structure seen for the displacement of the minor component $\kappa$-casein. This is consistent with the belief that the strongest network former in a mixture dominates failure of the mixture. However, the result is surprising because of the low levels of $\kappa$-casein present in 
sodium caseinate. The data suggests that on compression the 'weaker' components (the $\beta$ and $\alpha$-caseins) are preferentially displaced. Given the decrease in area occupied by the protein network as the surfactant domains expand this will result in the concentration of the $\kappa$-casein component. The suggestion is that, paradoxically, this results in the formation of a strong $\mathrm{K}$-casein network, which resists further displacement. The ability to visualise the networks allows such mechanisms to be proposed and tested.

Thus the modelling of protein isolates as simple protein mixtures provides a basis for understanding and manipulating their properties.

Model and real systems: The molecular models for displacement are based on AFM studies of interfacial structures sampled by LB methods. One might question whether the sampling procedure alters the interfacial structure? From the current AFM studies it is possible to predict the distribution of surfactant domain sizes as a function of added surfactant (Mackie et al, 2003). Thus it is possible to determine when the domains induced by non-ionic surfactant are large enough to be visible by Brewster Angle Microscopy (BAM). Combined AFM and BAM studies have confirmed (Mackie et al, 2001b) the presence of surfactant domains directly at the interface. In situ observations of protein displacement from a solid surface (Gunning et al, 1999) show that the domain structures observed by AFM cannot be attributed to drying artefacts. Finally, data on liquid lamellae, as models for foam drainage, show the presence of domains of different thickness when both protein and surfactant are present (Clark et al, 1990; Wilde et al, 1993; 2004). Fluorescence recovery after photobleaching measurements show different rates of diffusion (Clark et al, 1990; 1994; Wilde et al, 1993; 2004) consistent with the thinner 'surfactant' domains containing highly mobile molecules, and the thicker 'protein' domains containing immobile molecules as expected for a protein network. It would still be useful to demonstrate that orogenic displacement also occurs on finite-sized, curved surfaces, representing the spherical oil droplets present in oil-in-water emulsions. It has proved possible to use AFM to measure the interaction between oil droplets in aqueous media (Gunning et al, 2004c). The interfacial structure of the droplets attached to the cantilever and the substrate surface can be controlled. As the droplets approach each other the droplets deform. By monitoring the deformation as a function of added surfactant it has been possible to follow the displacement of protein from the interfaces. Such studies were found to be consistent with orogenic displacement of protein from the oil-water interface (Gunning et al, 2004c). Furthermore, the studies showed that, at least for the droplet sizes studied, the deformation of protein-coated droplets was dictated by the surface elasticity of the protein film, rather than the interfacial tension and the internal Laplace pressure within the droplets.

Knowledge of the competitive displacement mechanisms allows rational modification of the interfacial structure to improve functionality. Because the molecular displacement mechanism is generic these strategies can be employed for a range of food systems produced by the baking, brewing and dairy industries. A variety of intervention strategies have been suggested or implemented. Broadly speaking it is possible to strengthen the protein network to resist failure, or to remove the mobile small molecules that breakdown the protein network.

Understanding the role played by proteins makes it easier to investigate the mode of action of 'surface-active' polysaccharide. As an example consider sugar beet pectin: sugar beet pectin is unusual in that, unlike normal citrus or apple pectin, it can function as an emulsifier. Sugar pectin contains higher levels of protein than most pectin and the protein component is difficult to remove; suggesting that it may be covalently linked to the pectin. AFM images 
(figure 6) of 'sugar beet pectin' reveal that about 33\% of the molecules are pure polysaccharide chains (Kirby et al, 2006). The majority of the chains are linear but a small fraction have branched polygalacturonic acid backbones. More importantly, about $67 \%$ of the molecules are protein-polysaccharide complexes, with the protein attached at one end of the pectin chain (Kirby et al, 2006). These images immediately suggest why these extracts should function as good emulsifiers. The protein should be able to adsorb at the oil-water interface and form a network. This network would than be encased in a sheath of carbohydrate which would protect the protein network from displacement. In addition steric interactions between the carbohydrate-coated droplets would enhance stability against coalescence. Again the ability to visualise structures allows mechanisms to be proposed and tested, and promises to allow enhancement of the properties of the material as an emulsifier, or the design of new emulsifiers based on this structure.
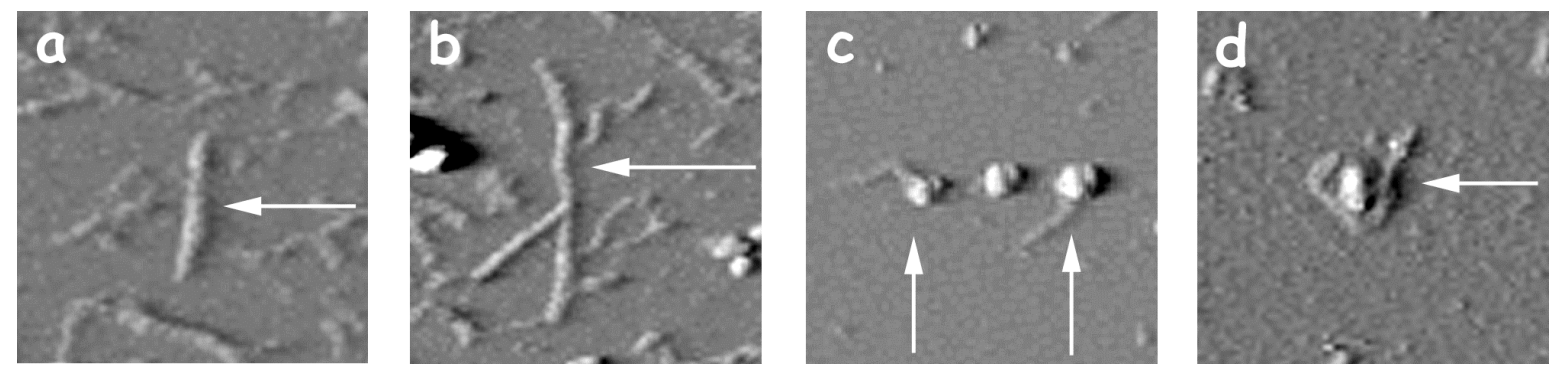

Figure 6. AFM images (230 x $230 \mathrm{~nm})$ of (a) isolated, uncomplexed linear (helical) pectin; (b) uncomplexed, branched pectin chains; (c \& d) protein-polysaccharide complexes, with extended chains (c) and chains coiled around the protein (d). Examples of the structures are arrowed.

\section{STARCH}

Sample preparation and imaging: The methodology for imaging the internal structure of starch can best be illustrated on pea and mutant pea starch granules. Granules were encased in rapid-set Araldite ${ }^{\mathrm{TM}}$ (Bostik Ltd) which has been shown (Ridout et al, 2002) not to penetrate into the interior of the granule. A drop of the Araldite ${ }^{\mathrm{TM}}$-starch granule mixture was allowed to set on a mica surface, the underside of which was then attached to the surface of an aluminium specimen stub using double-sided Sellotape ${ }^{\mathrm{TM}}$. The top surface of the block was honed down with a freshly-prepared glass knife to produce a flat, shiny surface consisting of starch granules with cut surfaces exposed. The mica was cleaved to produce a dry flat base that could be put onto a microscope slide, so that the cut face could be imaged by AFM.

To prepare sections of encased starch the starch granules were mixed with the rapid-set Araldite $^{\mathrm{TM}}$ and then transferred to a mould (Baker, Miles \& Helbert, 2001; Ridout et al, 2003). Rapid-set Araldite ${ }^{\mathrm{TM}}$ sets in about 2 minutes and becomes hard within 24 hours, at which time it becomes possible to cut sections. Sections, of nominal thickness $1.5 \mu \mathrm{m}$, were cut onto water using glass knives and an Ultramicrotome (Ultracut E, Reichert-Jung). The sections were transferred immediately to a drop of distilled water on a glass slide. Individual sections were quickly moved from the edge of the drop onto an area of dry glass. The slide was then left to dry at room temperature. Although it was possible to cut thinner or thicker sections, a thickness of about $1.5 \mu \mathrm{m}$ maintained the integrity of the starch granules during sectioning.

The cut faces of the blocks and sections of encased starches are quite rough. An easy way to visualise the surfaces by AFM is to collect error signal mode images. The AFM is operated in 
the normal dc contact imaging mode. Because the samples are rough there is generally insufficient time for the feedback circuit to adjust the error signal at each image point to zero. Thus images are generated by amplifying the residual error signal. The rough samples consist of a broad background with the important surface detail superimposed on this background. The feedback loop effectively filters out, or effectively subtracts the background, revealing the detailed surface structure. The change in height in the image is determined by surface roughness and is representative of surface topography. However, the height (grey levels) in the image represents changes in force and is not a true measure of surface topography or height. The error signal mode image reveals what the surface will look like: it is then possible to process the true topography images to generate images of the details of the surface structure.
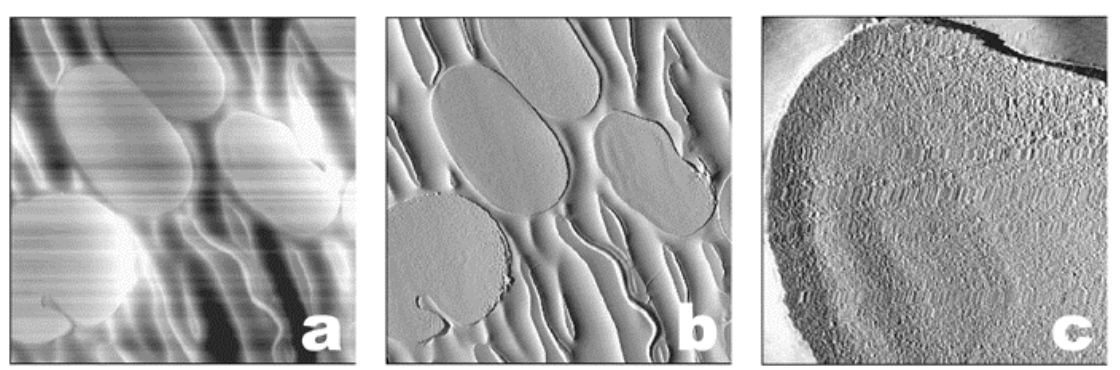

Figure 7. AFM images of unwetted, cut faces of encased pea starch granules. (a) Topography and (b \& c) error signal mode images. The scan sizes are (a) \& (b) $80 \times 80 \mu \mathrm{m}, \&$ (c) 8.7 x $8.7 \mu \mathrm{m}$.

Contrast mechanisms: Figure 7a shows a topographical image of the cut faces of an unwetted encased pea starch sample. The granules can be distinguished from the surrounding resin mainly due to the knife judder present in the images of the resin material. However, there are no internal structural features visible on the exposed surfaces of the starch granules. The samples are fairly rough and, in general, it is often difficult to visualise fine detail in the images of these rough surfaces. As described earlier it has become common practice to use the residual error signal as a basis for visualizing detail in the surface structure on such rough samples.
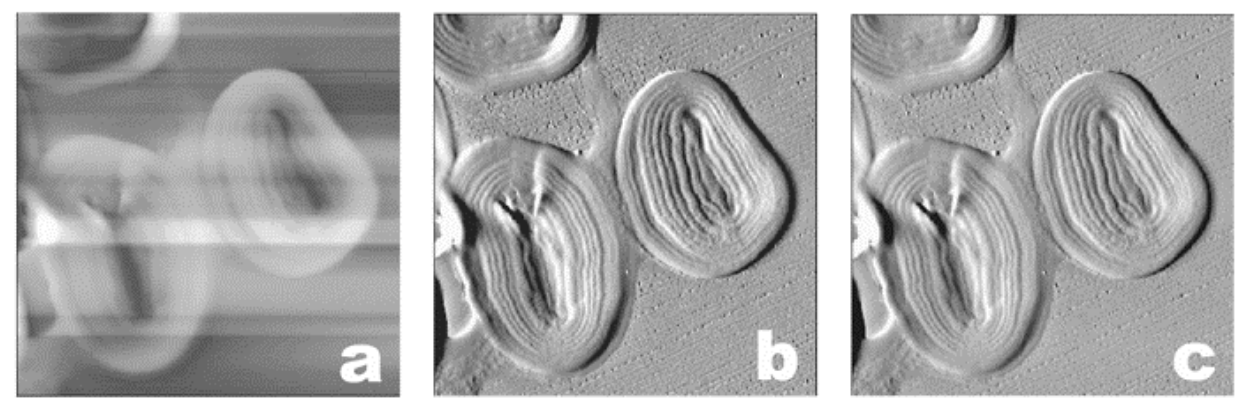

Figure 8. AFM images of wetted pea encased starch granule sections. (a) Topography, (b) error signal mode, and (c) left-shaded topography images. Scan sizes are 35.5 x $35.5 \mu \mathrm{m}$.

Error signal mode images of cut faces of the unwetted encased pea starch are shown in figures $7 \mathrm{~b} \& \mathrm{c}$. Although there is a difference in contrast between the granule and the encasing resin, presumably due to the different elastic moduli of the materials, the exposed face of the granule is still seen to be featureless. However, AFM images of sectioned, encased starch, 
which have been wetted during sample preparation, reveal considerable detail about the internal structure of the granules (figure 8).

The topography image (figure 8a) shows that the granule surface itself is quite rough. The exposed surface of the granule appears to bulge out above the surface of the surrounding resin at the edges of the granule, but it is dark and collapsed at the centre of the granule. Even in these rough samples it is possible to discern a concentric banded structure within the granule. This is more clearly evident in the error signal mode images (figure $8 \mathrm{~b}$ ). Comparison of figure $8 \mathrm{~b}$ and figure $8 \mathrm{a}$ suggest that the banding results from a relatively low-amplitude ripple superimposed on the high-amplitude bowed structure of the exposed face of the granule. This is confirmed by the images in figure 8c where the surface ripple has been emphasized by shading the topographic images.

The band-like structure seen in figure 8 is reminiscent of the familiar growth ring structure of the starch granule. The important question is why is this structure seen in the sectioned material (figure 8) but not on the cut faces (figure 7) of the block? In both cases the starch granules are encased in Araldite ${ }^{\mathrm{TM}}$ resin. However sectioning exposes the underside of the sections to water and the effect of this hydration step on image contrast has to be considered. This can be investigated by comparing images of cut faces of encased starch before (figure 7) and after exposure to water (figure 9).
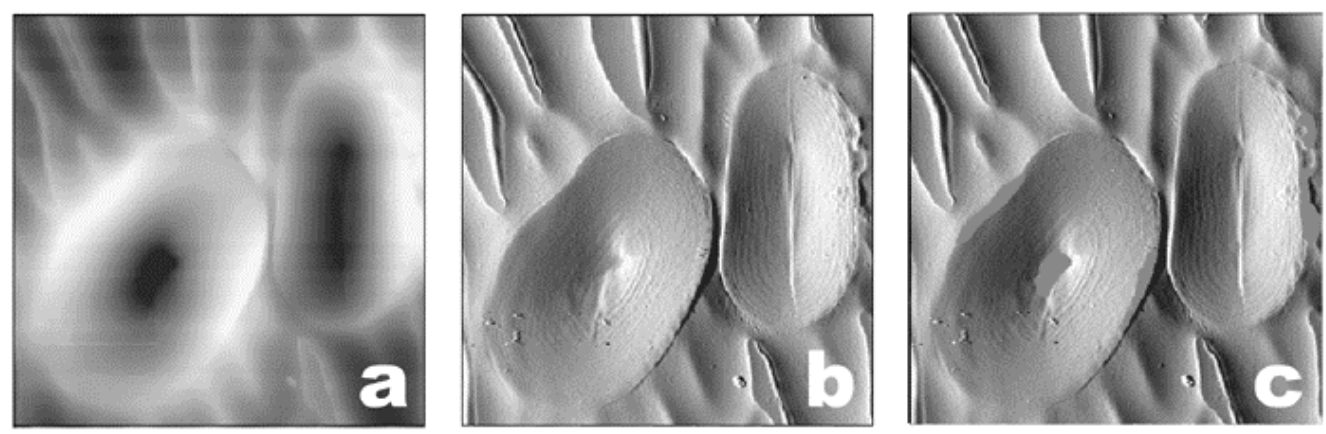

Figure 9. AFM images of wetted cut faces of encased pea starch granules. (a) Topography, (b) error signal mode and (c) left-shaded topography images. Scan size 29.1 x $29.1 \mu \mathrm{m}$. After wetting the samples have been dried extensively over $\mathrm{P}_{2} \mathrm{O}_{5}$.

AFM mages shown in figure 9 were generated by depositing a drop of distilled water onto the cut face of an unwetted cut face, removing the excess water by blotting, storing the sample in air at room temperature and then in a desiccator over $\mathrm{P}_{2} \mathrm{O}_{5}$ for 3 months. The detail in figure 9 appears once the cut faces have been wetted, and the induced changes are irreversible.

It is clear that the topography of the cut face has changed on wetting. The new images now resemble those seen with the sectioned material (figure 8). The topographic images (figure 9a) show that there is a large variation in height, of the order of $>1 \mu \mathrm{m}$, across the exposed face of the granule. This must be due to water being absorbed into the granule, and the subsequent swelling of the exposed granule surface out of the top surface of the block during wetting (Ridout et al, 2004; Morris et al, 2005).

It has been reported previously that growth rings are absent, or infrequently observed in AFM images of sectioned embedded starch material (Baker, Miles \& Helbert, 2001; Ridout et al, $2002 ; 2003)$. In these cases the resins used penetrated throughout the interior of the granule. 
For melamine resins such as Nanoplast ${ }^{\mathrm{TM}}$ it was noted that the curing process may also degrade amylosic chains within the granule, and may even induce crystallisation of fragmented chains (Ridout et al, 2002). No equivalent banding was seen in the AFM images. The studies described above provide an explanation for these observations. Contrast in the AFM images depends on the swelling of the hydrated regions. Swelling introduces height variations across the exposed faces of the granules and the more hydrated regions will be softer and easier to compress, both factors contributing to the contrast in the topography and error signal mode images. The setting of the melamine resin generates a tightly cross-linked network which will limit swelling of the granule on hydration and inhibit contrast in the AFM images.

Growth rings and blocklets: At higher resolution, where the region of the exposed surface of the granule is smaller and flatter, it is possible, if the wetting process is optimized, to visualise the internal structure of the starch granule in more detail. The banding is found to consist of swollen and less-swollen bands within the granules. Distributed throughout the granules are spheroidal objects identified with the blocklet structures first detected by TEM studies. These blocklets are believed to be the small parcels of crystal and amorphous lamellae formed by the branched amylopectin molecules. There have been suggestions that each blocklet may represent what becomes an individual amylopectin molecule on extraction of the starch polysaccharides. The bright and dark bands are clearly related to the growth ring structure of the granule. The AFM images reveal bright (swollen) bands and dark (lessswollen) bands. As can be seen in figure 10 the dark bands are discontinuous, containing bright swollen regions and dark unswollen patches which show no internal detail.

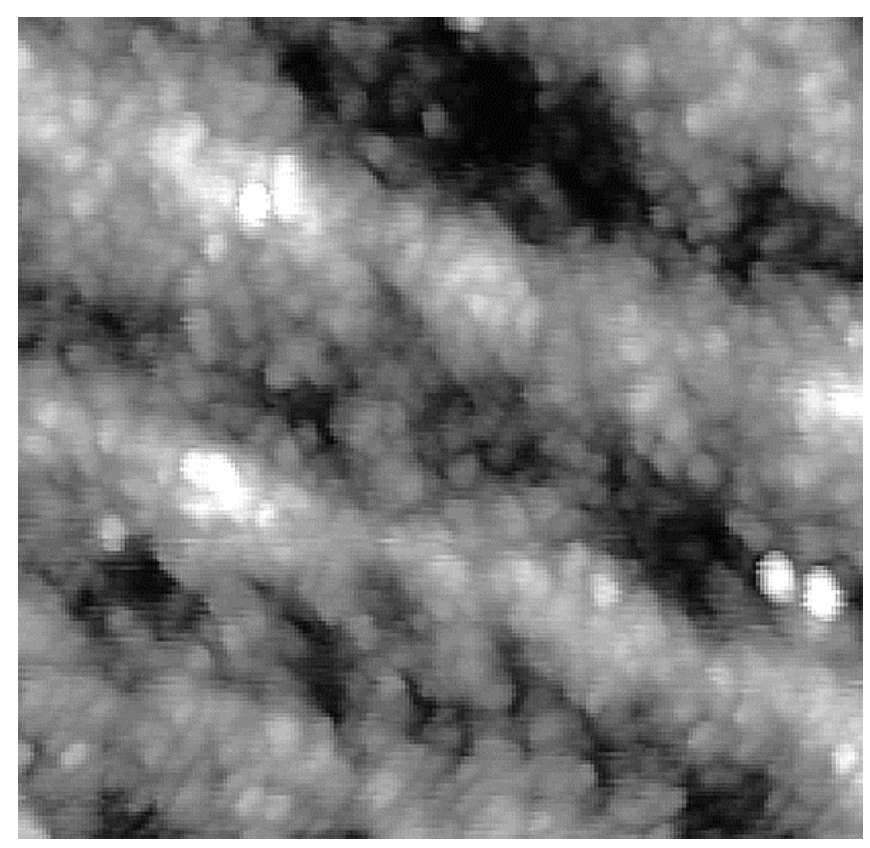

Figure 10. Higher resolution topography AFM image of the internal structure of a pea starch granule. Image size $1.36 \times 1.36 \mu \mathrm{m}$. The image shows blocklets and the banding pattern within the granule.

The swelling of the exposed faces of the granules is considered to arise from preferential wetting of the amorphous regions within the granules. These are believed to be the matrix, largely composed of amylose, in which the blocklets are embedded. On such a model the largely unswollen patches within the dark bands might be considered to be regions where the amylose concentration is lower and the swelling less significant. However, the images clearly 
show that the whole granule is partially crystalline and that the alternate bands differ in their level and distribution of amorphous material. This is in conflict with the presently accepted view that the granules contain alternate growth rings that are either crystalline or amorphous.
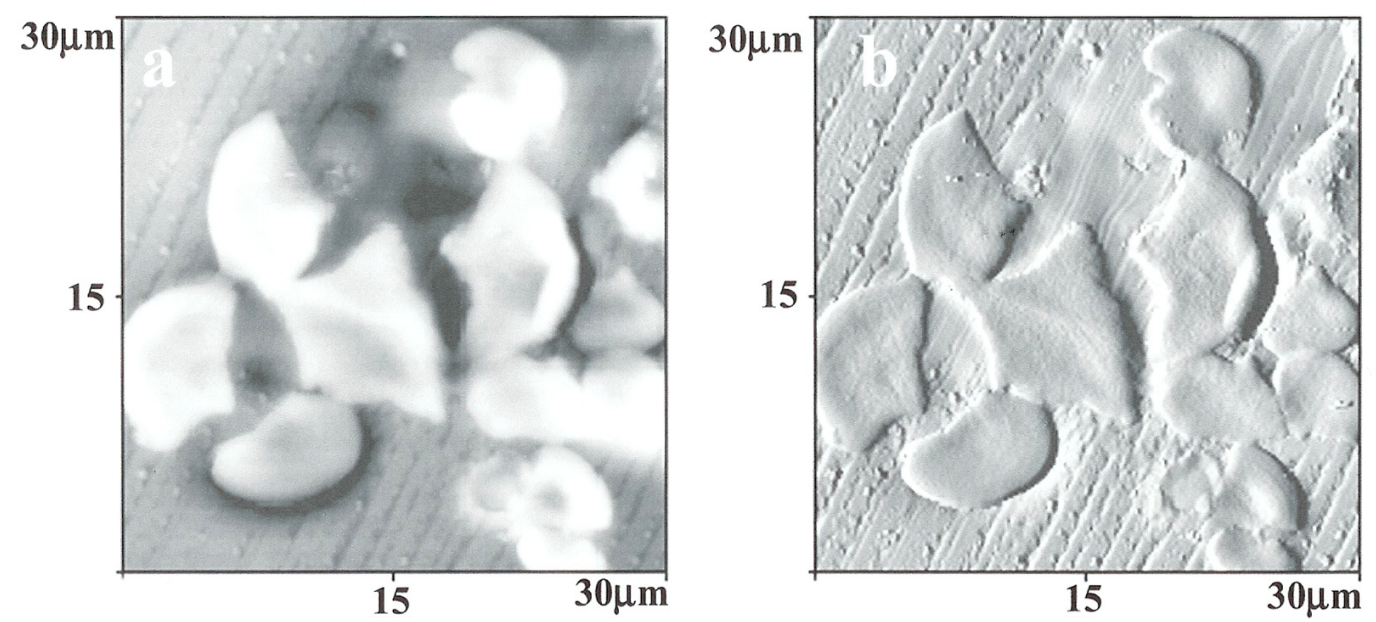

Figure 11. AFM images of sectioned, encased $r$ mutant pea starch granules. (a) Topography images and (b) error signal mode images.
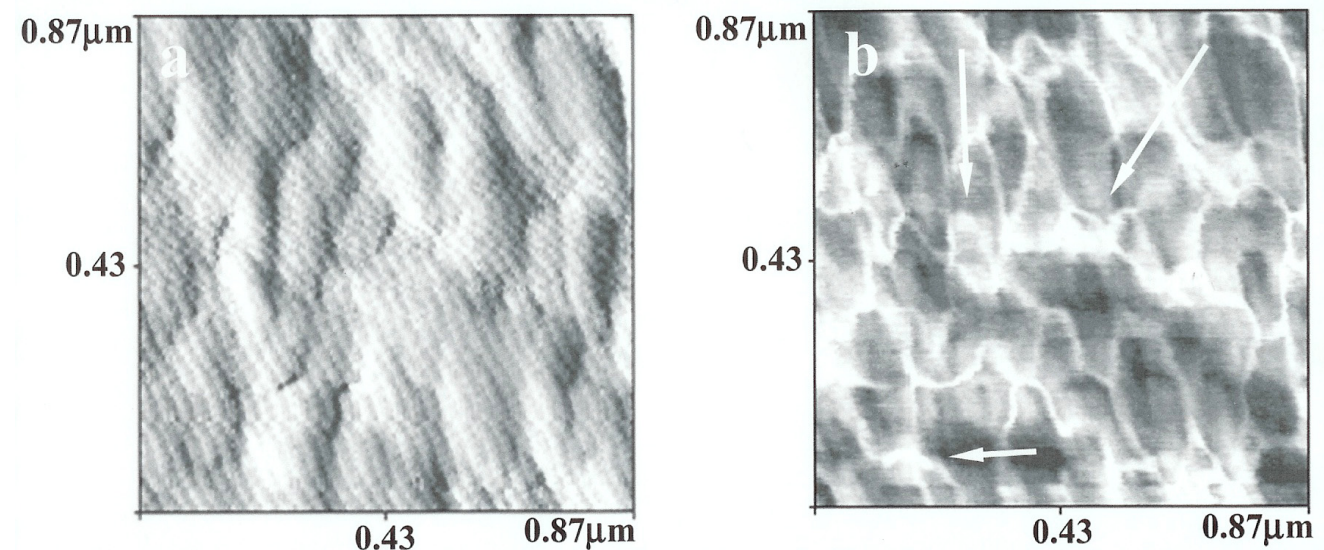

Figure 12. AFM images of the internal structure of sectioned, encased $r$ mutant pea starch granules. (a) Error signal mode image and (b) force modulation image. The arrows indicate a hard network structure permeating throughout the granule.

High-amylose pea starches: It is instructive to use the methodology developed to image the interior structure of starch granules, in order to investigate how mutations in starch biosynthesis alter granule structure and function. This can best be done using isogenic starch mutants, where the structural changes can be attributed to mutations in single genes in an otherwise constant genetic background. Certain high-amylose pea starches show distinct changes in functional properties (Bogracheva et al, 1999; Ridout et al, 2003; 2004). The $r$ mutant results from a mutation in the starch branching enzyme A. This leads to decrease in the amylopectin content of about $50 \%$ without any significant reduction in crystallinity within the granule. The crystal polymorph changes from C-type to essentially pure B-type and lintnerisation studies suggest that the crystalline component contains higher molecular weight amylosic chains, in addition to the expected short branches of the amylopectin molecules. The gelatinisation behaviour is modified, becoming broadened, and complete swelling and gelatinisation only occurs $>100^{\circ} \mathrm{C}$. AFM images of the granules (figure 11) show fractured 
and broken granules lacking any visible growth rings. The topography images are quite flat showing little swelling of the exposed face of the granules.

At higher resolution, error signal mode images (figure 12a) show what appears to be a 'blocklet' structure within the granule. However, the blocklets appear larger in size than those observed in the wild-type starch. Because the sample is a thin section supported on a hard background (substrate) it is possible to attempt to map the hardness of the sample using force modulation. In this technique the cantilever-probe assembly is oscillated and the image generated from the initial slope of the force-distance curves. This effectively maps the modulus of the surface, with the hardest regions appearing bright. These studies, although very difficult and subject to artefacts, do seem to indicate (figure 12b) the presence of a novel thin network structure permeating throughout the granule (Ridout et al, 2003; 2004). Thus the image in figure 12a may reflect the pore size of this network rather than individual blocklets. Such a network would account for the reduced swelling of the sections. If the network is partially crystalline it would account for the fragility and fracture of the granules. It has been suggested (Ridout et al, 2003; 2004) that the network is composed of partially crystalline amylosic chains and that the varying length of the chains forming the crystallites results in the broadened gelatinisation and restricted swelling of the intact granules. The presence of such networks would not only influence the functional properties of the starches but would influence the digestibility of the cooked starches, changing their nutritional value.

\section{CONCLUSIONS}

It has been demonstrated that the use of AFM can generate new information on the molecular heterogeneity of food systems. This knowledge has provided new understanding which can be exploited to manipulate rationally the selection and processing of food materials.

\section{ACKNOWLEDGEMENTS}

The research described in this article was supported by the BBSRC through core funding and responsive mode grants (D14067, D13192, D11145). The apparatus used in the study was purchased through funding from the BBSRC Bioimaging initiative. Part of the research on starch was funded through the EU (Framework 4 FAIR (CT98-3527)). The research on interfaces was in collaboration with Alan Mackie and Peter Wilde, with the AFM studies being developed and performed by Paul \& Patrick Gunning and Nicola Woodward. The AFM measurements on starch were performed by Mike Ridout, Mary Parker and Andrew Kirby in collaboration with Cliff Hedley and Tanya Bogracheva at the John Innes Centre, Norwich, UK.

\section{References:}

Baker AA, Miles MJ, Helbert W. (2001). Carbohydr. Res. 330, 249.

Bogracheva TY, Cairns P, Noel TR, Hulleman S, Wang TL, Morris VJ, Ring SG, Hedley CL. (1999). Carbohydr. Polym. 39, 303.

Buléon A, Colonna P, Planchot V, Ball S. (1998). Int. J. Biol. Macromolecules 23, 85.

Clark DC, Coke M, Mackie AR, Pinder AC, Wilson DR. (1990). J. Colloid \& Interface Sci. 138, 207.

Clark DC, Mackie AR, Wilde PJ, Wilson DR. (1994). Faraday Discussions 98, 253.

Gallant DJ, Bouchet B, Baldwin PM. (1997). Carbohydr. Polym. 32, 177. 
Gunning AP, Wilde PJ, Clark, DC, Morris VJ, Parker ML, Gunning PA. (1996). J. Colloid \& Interface Sci. 183, 600.

Gunning AP, Mackie AR, Wilde PJ, Morris VJ. (1999). Langmuir 15, 4636.

Gunning AP, Mackie AR, Kirby AR, Morris VJ. (2001). Langmuir 17, 2013.

Gunning PA, Mackie AR, Gunning AP, Wilde PJ, Woodward NC, Morris VJ. (2004a). Food Hydrocolloids 18, 509.

Gunning PA, Mackie AR, Gunning AP, Woodward NC, Wilde PJ, Morris VJ. (2004b). Biomacromolecules 5, 984.

Gunning AP, Mackie AR, Wilde PJ, Morris VJ. (2004c). Langmuir 20, 116.

Helbert W, Chanzy H. (1996). Stärke 48, 185.

Kirby AR, MacDougall AJ, Morris VJ. (2006). Food Biophysics 1, 51.

Mackie AR, Gunning AP, Wilde PJ, Morris VJ. (1999). J. Colloid \& Interface Sci. 210, 157.

Mackie AR, Gunning AP, Wilde PJ, Morris VJ. (2000a). Langmuir 16, 2242.

Mackie AR, Gunning AP, Wilde PJ, Morris VJ. (2000b). Langmuir 16, 8176.

Mackie AR, Gunning AP, Ridout MJ, Wilde PJ, Morris VJ. (2001a). Langmuir 17, 6593.

Mackie AR, Gunning AP, Ridout MJ, Wilde PJ, Patino JR. (2001b). Biomacromolecules 2, 1001.

Mackie AR, Gunning AP, Wilde PJ, Morris VJ, Pugnaloni LA, Dickinson E. (2003). Langmuir 19, 6032.

Morris VJ. (2004). Trends Food Sci. \& Technol. 15, 291.

Morris VJ, Ridout MJ, Parker ML. (2005). Progr. Food Biopolymer Res. 1, 28.

Ridout MJ, Gunning AP, Wilson RH, Parker ML, Morris VJ. (2002). Carbohydr Polym. 50, 123.

Ridout MJ, Parker ML, Hedley CL, Bogracheva TY, Morris VJ. (2003). Carbohydr. Res. $338,2135$.

Ridout MJ, Parker ML, Hedley CL, Bogracheva TY, Morris VJ. (2004). Biomacromolecules $5,1519$.

Waigh TA, Perry P, Riekel C, Gidley MJ, Donald AM. (1998). Macromolecules 31, 7980.

Waigh TA, Kato KL, Donald AM, Gidley MJ, Clarke CJ, Riekel C. (2000). Stärke 52, 450.

Wilde PJ, Clark DC. (1993). J. Colloid \& Interface Sci. 155, 48.

Wilde PJ, Mackie AR, Husband FA, Gunning AP, Morris VJ. (2004). Adv. Colloid \& Interface Sci. 63,108.

Woodward N., Wilde PJ, Mackie AR, Gunning AP, Gunning PA, Morris VJ. (2004). J. Agric Food Chem. 52, 1287. 